\title{
Evaluación de la Analgesia Epidural en los Resultados Perinatal y Obstétrico
}

\author{
INSTITUTO MATERNO INFANTIL BOGOTA 1988
}

\author{
Rodrigo Díaz Llano, MD, MSP*, Germán Sandoval Vélez, MD** \\ Pedro J. Sánchez Villamizar, MD***
}

\section{INTRODUCCION}

El uso de analgesia durante el trabajo de parto (T. de P.) y el parto, se ha ido imponiendo probablemente por la mayor aceptación entre los médicos de que el parto es un proceso doloroso y que el dolor no es indispensable para que este evento transcurra normalmente. Inclusive, el alivio del dolor durante este período puede ser benéfico por cuanto disminuye las concentraciones plasmáticas de catecolaminas $(1,2)$, y parece que el incremento de catecolaminas puede causar depresión del feto y del neonato, por vasoconstricción de los vasos ute$\operatorname{rinos}(3,4)$.

En nuestro medio, un factor que podría haber contribuido al uso y difusión de la analgesia epidural, es la aceptación de su aplicación de rutina en la práctica privada de la obstetricia, al menos en Bogotá.

En el Instituto Materno Infantil (IMI) también se ha incrementado paulatinamente el uso de analgésicos, siendo la analgesia epidural uno de los métodos utilizados. Existen algunos informes que reportan compromiso neonatal debido a su uso (5), como también otros que aseguran su inocuidad cuando es bien conducida $(6,7)$. Aun cuando existe algún debate sobre la conveniencia o no en el uso rutinario de la epidural durante el T. de P. y se afirma en la literatura que muchos obstetras inte-

\footnotetext{
* Profesor Asistente, Ginecología y Obstetricia Facultad de Medicina, Universidad Nacional de Colombia.

** Instructor Asociado, Anestesiología Facultad de Medicina. Universidad Nacional de Colombia.

*** Instructor Asociado, Anestesiología Facultad de Medicina. Universidad Nacional de Colombia.
}

rrumpen esta analgesia en el segundo período del T. de P. con la intención de mejorar o acortar el mismo, esto no parece ser una práctica sensata (8).

En este estudio, se evaluaron los partos atendidos con analgesia epidural, comparándolos con partos sin analgesia, observando el Apgar de los recién nacidos (R. N.) y la frecuencia de instrumentación del parto.

\section{METODOS}

El IMI es un hospital universitario, que intenta recibir pacientes obstétricas de alto riesgo, seleccionadas en los centros de salud de su área de influencia. En general, ellas son pacientes de bajos estratos socioeconómicos, sin ningún tipo de protección de la seguridad social y que usualmente no han recibido educación ni preparación psicológica para el parto. Este estudio se circunscribe a los partos ocurridos entre el 1 de enero y el 31 de diciembre de 1988 .

En 1988 se presentaron en el IMI 9.929 nacimientos. De ellos, 9.806 fueron nacidos vivos y 278 nacidos muertos. Se practicaron 2.489 operaciones cesáreas. Se observaron 73 partos múltiples (uno triple, los demás gemelares). En este año, se aplicaron 588 anestesias epidurales, de las cuales 239 fueron destinadas para las cesáreas (las cesáreas restantes recibieron otros tipos de anestesia).

De las pacientes que terminaron su parto por vía natural, recibieron analgesia c sidural 349. Es interesante anotar que de ellas, 290 se aplicaron en el día y 59 en la noche, aun cuando la distribución de los partos es sustancialmente igual en la noche como en el día. 
Para evaluar los resultados de la analgesia epidural en el parto normal, se revisaron los casos de las madres a quienes se les aplicó esta analgesia. Se excluyeron del estudio las madres que fueron sometidas a operación cesárea, como también aquellas con factores de riesgo para morbimortalidad perinatal o materna no relacionado con la analgesia obstétrica (toxémicas, embarazos múltiples, muertes o sufrimientos fetales agudos o crónicos detectados antes de ordenar la aplicación de la analgesia). Además, se excluyeron los casos de R. N. con pesos inferiores a $2.500 \mathrm{~g}$ o superiores a $3.800 \mathrm{~g}$ para excluir otros factores de riesgo, como prematurez, retardo de crecimiento intrauterino, macrosomía, prolongación del segundo período por desproporción fetopélvica (características independientes de la analgesia). Con estas exclusiones se pretendió evitar asociaciones no causales, introducidas por factores de confusión.

Para identificar el grupo de comparación, se revisaron los libros de registro de nacimientos del IMI seleccionándose las pacientes con parto vaginal y que recibieron analgesia epidural. De la misma manera, se identificó un grupo de posibles controles, correspondientes a pacientes con parto vaginal y que no recibieron analgesia epidural. Utilizando los datos de la historia clínica, se descartaron las pacientes de ambos grupos que poseyeran los factores de exclusión anotados anteriormente y se estableció la paridad. Cada caso fue apareado con la siguiente paciente control en el registro de nacimientos, que tuviera igual paridad.

Los datos de Apgar al minuto del R. N., y la utilización o no de instrumentación quirírgica del parto - forceps o espátulas (9)-, se obtuvieron del libro de registro de nacimientos.

Los datos fueron procesados manualmente y se analizaron calculando la distribución del Apgar y de la instrumentación del parto, en los casos con y sin analgesia epidural. Se utilizó una prueba de chi cuadrado para establecer la significancia estadística de las diferencias observadas. Para poder aplicar esta prueba, no se tuvieron en cuenta las casillas con ceros (APGAR 1-3) que fue igual en los dos grupos.

En el IMI, se aplica la analgesia epidural en la fase activa del T. de P. y en general cuando el cuello ha llegado a unos 6 ó $7 \mathrm{~cm}$ de dilatación. En la técnica de aplicación se aborda el espacio epidural lumbar por entre los espacios intervertebrales $\mathrm{L}$ 3-4 ó L 4-5. Casi siempre se deja puesto un catéter para aplicación de nuevas dosis. Se utiliza como agente anestésico la bupivacaina (Marcaina simple) en concentraciones de 0,125 ó de $0,25 \%$ y se aplican dosis de 12,5 ó de $25 \mathrm{mg}$ (volumen $=10$ c.c.). Previamente se administra a la paciente 1.000 c.c. de solución de Ringer, en un lapso no mayor de 20 minutos antes de la aplicación de la analgesia epidural.

\section{RESULTADOS}

De las 349 pacientes que recibieron analgesia epidural, y que terminaron por parto natural, se excluyeron 128 por las razones establecidas. Por lo tanto la muestra de estudio quedó conformada por 211 pacientes y otro número idéntico de madres conformaron el grupo de comparación (no recibieron analgesia epidural).

El Cuadro 1 muestra las distribuciones de puntajes Apgar que obtuvieron al minuto los R. N. de madres que recibieron analgesia epidural, comparándolos con los de las madres que no recibieron analgesia. Puede observarse que los resultados son semejantes, y en efecto, no resultó diferencia estadísticamente significativa.

\section{CUADRO 1}

\section{COMPARACION DE RESULTADOS DE APGAR}

\begin{tabular}{|l|c|c|c|c|c|}
\hline EPIDURAL APGAR & $1-3$ & $4-5$ & $6-7$ & $8-10$ & TOTAL \\
\hline CON ANALGESIA & 0 & 2 & 40 & 179 & 221 \\
\hline SIN ANALGESIA & 0 & 1 & 31 & 189 & 221 \\
\hline
\end{tabular}

$$
C h i^{2}=1.75(P=0.418) \text {. }
$$

En el Cuadro 2 se presenta la frecuencia de instrumentación del parto en los dos grupos de pacientes. En éstos sí se aprecia una diferencia notable; en efecto, el parto fue instrumentado con una frecuencia 5 veces mayor en las pacientes que recibieron analgesia epidural, en comparación con las que no la recibieron (diferencia altamente significativa).

\section{CUADRO 2}

FRECUENCIA DE INSTRUMENTACION DEL PARTO EN PACIENTES CON Y SIN ANALGESIA EPIDURAL

\begin{tabular}{|l|c|c|c|c|c|}
\hline \multirow{2}{*}{ TIPO DE } & \multicolumn{2}{|c|}{ INSTRUMENTADO } & \multicolumn{2}{c|}{ NO INSTRUMENTADO } & \multirow{2}{*}{ TOTAL } \\
\cline { 1 - 7 } EPIDURAL & F & $\%$ & F & $\%$ & \\
\hline SIN ANANALGESIA & 156 & 70.6 & 65 & 29.4 & 221 \\
\hline
\end{tabular}

$\mathrm{Cni}^{2}=144.8$ $P<0.000$ 


\section{DISCUSIION}

Si bien es cierto que el Apgar no es el único indicador del estado del R. N., es un indicador aceptado de la situación del feto durante el proceso del T. de P. y el parto. De acuerdo con los resultados de este estudio, se puede suponer que la administración de analgesia epidural durante el T. de P. y el parto, no modifica las condiciones del feto y por lo tanto esta no sería una razón para no administrar la analgesia a la madre, a partir del momento en que el cuello alcanza una dilatación de unos 6 ó $7 \mathrm{~cm}$. Tampoco parece razonable suspender la analgesia epidural en el segundo período del parto. Esta práctica nunca se ha utilizado en el IMI, y los resultados son los descritos.

De otra parte, parece que las pacientes que reciben esta analgesia, requieren con más frecuencia instrumentación de su parto. Podría pensarse que esto se debiera a la inhibición del reflejo del pujo por el efecto de la epidural. Sin embargo existe un estudio que muestra menor utilización de instrumentación del parto con analgesia epidural (10). La mayor proporción de instrumentación observada en este estudio, podría explicarse (además de la señalada inhibición del reflejo del pujo), por el interés de entrenamiento de los Residentes. Cualquiera que pudiera ser la explicación de este hallazgo, lo que parece cierto, es que el mayor uso de instrumentación del parto, tampoco ejercería efectos indeseables sobre los resultados perinatales (11).

\section{CONCLUSIONES}

Parece conveniente aliviar el dolor de las contracciones uterinas durante el T. de P. La analgesia epidural manejada con cuidado (evitando la hipotensión secundaria a vasodilatación, administrando líquidos endovenosos), parece ser el método analgésico adecuado, por cuanto no ejerce los efectos sistémicos de los analgésicos narcóticos (12).

De acuerdo con los hallazgos, no hay diferencia en los resultados perinatales en los R. N. de las madres que recibieron analgesia epidural, y en las que no recibieron ningún tipo de analgesia durante el T. de P. y el parto. Por otra parte, sí se encontró una diferencia importante en la forma de terminación del parto, por cuanto la proporción de instrumentación fue 5 veces mayor en las pacientes que recibieron epidural, aun cuando esto tampoco parece afectar los resultados perinatales.

\section{RESUMEN}

Con el propósito de evaluar los resultados perinatales y obstétricos (Apgar e instrumentación del parto) de las pacientes que reciben analgesia epidural durante el trabajo de parto y el parto, se observaron los resultados obtenidos por $221 \mathrm{pa}-$ cientes que recibieron esta analgesia, y que no tenían factores de riesgo materno-fetal. Este grupo fue comparado con otro de similares características y que tuvieron el parto sin ningún tipo de analgesia en el mismo período. No se encontraron diferencias en los resultados de Apgar en los recién nacidos de ambos grupos. Por otra parte, las pacientes que recibieron analgesia epidural, requirieron instrumentación del parto en una proporción 5 veces mayor, que las que tuvieron su parto sin analgesia.

PALABRAS CLAVES: analgesia epidural, Apgar, instrumentación del parto.

\section{BIBLIOGRAFIA}

1. ABBOUD, T. K.; ARTAL, R.; SARKIS, F.; KAMMULA, R. K. Sympatho-adrenal activity: maternal, fetal and neonatal responses after epidural anesthesia in pre-eclamptic patient. Am J Obstet Gynecol 1982; 144: 524-527.

2. SHNIDER, S.M.; ABBOUD, T.K.; ARTAL, R.; HENRICKSEN, E. H. ; STEFANI, S. J. ; LEVINSON, G. Maternal catecholamines decrease during labor after lumbar epidural anesthesia. Am J Obstet Gynecol 1983; 147: 13-15.

3. SHNIDER, S. M. WRIGHT, R. G. LEVINSON, G. et al. Uterine blood flow and plasma norepinephrine changes during maternal stress in the pregnant ewe. Anesthesiology 1979; 50: 524-527.

4. MYERS, R. E. Maternal psychological stress and fetal asphyxia: a study in the monkey. Am J Obstet Gynecol 1975; 122: 47-59.

5. SCANLON, J. W.; BROWN, W. U.; WEISS, J. B.; ALPER, M. H. Neurobehavioral responses of newborn infants after maternal epidural anesthesia. Anesthesiology 1974; 40: 121-128.
6. ABBOUD, T. K. et al. Lack of adverse neonatal neuro behavioral affects of lidocaine. Anesth Analg 1983;62: 473-475.

7. DIAZ, J. ; GONZALEZ, I. ; FRANCO, G. Anestesia Peridural continua en la inducción y conducción del Parto. Rev. Col. Obst. Gin. 1970; 21 : 399-404.

8. CHEEK, T. G.; GUTSCHE, B. B. Analgesia epidural en el trabajo de parto y parto vaginal. Clínicas Obst. Ginec. 1987; 3 : 487-500.

9. VELASCO, A. Espátulas para la extracción fetal, un nuevo instrumento obstétrico. Rev. Col. Obst. Gin. 1975; 26: 17-32.

10. PHILLIPS, K. C.;THOMAS, T. A. Second stage of labour with or without extradural analgesia. Anesthesia 1983; 38: 972 976.

11. NISWANDER, K. R.;GORDON, M. Safety of the low-forceps operation. Am J Obstet Ginecol 1973; 117: 619-630.

12. SPIELMAN, F. J. Analgésicos sistémicos durante el trabajo de parto. Clínicas Obstet Ginec 1987; 3: 469-476. 